
v Table 1: Some of physics-related ERICS

\section{The ERIC}

Having ensured coherence in planning, next bottleneck was the timely setting-up by several Countries. The EU basic law could now be used. Upon proposal by ESFRI, the EU has approved in 2009 a "Regulation", valid as a national law in all EU member countries [4]. This allows the ERIC (European Research Infrastructure Consortium) as an International Organisation in the EU legal system, without a new international agreement each time. Members of an ERIC are Countries or IOs. Its setting-up requires minimum three EU Member or Associate Countries (at least one Member). Observer Countries or IOs are allowed without voting rights. Non-EU Countries can be members or observers accepting EU rules. 21 ERICs have been set-up so far [5]. The Statutes allow new members to apply, and numbers are increasing in most ERICs.

Each ERIC has the main purpose to establish and operate a RI on a non-economic basis. It may have limited economic activities provided they are closely related to its principal task and they do not jeopardise the achievement thereof. The common approach is open access for users who commit to publish their results. RIs with a limited capacity for access offer at least part of it based solely on the quality of the proposals selected by independent peer review, even coming from non-ERIC-member Countries. The policy is defined in a Charter at EU level [6].
The nature of the ERIC as an International Organisation allows some benefits, as the possibility to have own flexible procedures and tax exemptions for procurement for institutional use of the ERIC.

Compared to IOs, contracts for staff are within national laws (employment is not yet within the $\mathrm{EU}$ law), and funding commitments by the Members are less firm than for IOs which are based on parliamentary approval. Funding of time-limited projects is possible on the EU budget.

When setting-up an ERIC, participating Countries/ IOs can, in principle, agree on additional benefits. The EU Regulation defines, in fact, a standard template to be completed and adapted in a flexible way to fit a specific RI and its Members, in particular defining the scope of the ERIC's activities and the commitments for resources. Fixed are two Governing Bodies: the General Assembly of the ERIC Members (similar to Councils of IOs, the decision-making) and the Director or Board of Directors, executive body and legal representative of the ERIC, appointed by the Assembly. Additional consultative Bodies, as a Scientific/Technical Advisory and an Administrative/ Finance Committee are possible. Other aspects to be defined are voting rights and procedures, liabilities, selection and hiring of staff, etc.
NAME AND WEBSITE

NUMBER OF MEMBERS (M) AND OBSERVERS (0)

CERIC: ceric-eric.eu

$(8 \mathrm{M})$

EMSO: emso.eu

(8M)

EPOS: epos-ip.org

(12 M)

EURO ARG0: euro-argo.eu

$(12 M+10)$

EUBI: eurobioimaging.eu

$(13 M+10)$

EU SPALLATION SOURCE:

europeanspallationsource.se

$10 M+40$

INSTRUCT: instruct-eric.eu

$(14 M+10)$

JIVE: jive.nl

(7 M)
EXTENDED NAME AND RESEARCH SCOPE

Central European Research Infrastructure Consortium, offers integrated international access and operation of Analytical and Synthesis facilities for Materials and Biomaterials with focus on Centre East Europe.

European Multidisciplinary Seafloor and water column Observatory, a network of observatories installed in European seas providing key data on marine ecosystems, natural hazards and climate change.

European Plate Observing System will provide a better understanding of the physical processes controlling earthquakes, volcanic eruptions, tsunamis, tectonic movements and other geo-hazards.

Euro Argo is the European part of a global array of autonomous instruments deployed over the world ocean and reporting near-real time subsurface ocean properties to a wide range of users via satellite.

Euro-Biolmaging offers open access to imaging technologies, training and data services in biological and biomedical imaging.

European Spallation Source is the world's next-generation neutron source under construction in Lund, Sweden. A high-power linear proton accelerator will create neutron beams to probe structures and dynamics of materials.

INtegrated STRUCTural biology is a distributed research infrastructure for structural biology, and provides access to cutting edge instrumentation (ultra-high field NMR, super-resolution cryo-EM, X-ray, protein production etc.).

Joint Institute for VLBI will develop the most accurate timing distribution infrastructure in the world enabling cutting-edge applications of VLBI (very long baseline interferometry) and radio astronomy technologies.

Code: Environment, Biomedical, Physics and Engineering 
Compared to the IOs, the Members of the ERIC may delegate one or more "Representing Entities" as regards the exercise of specified rights and the discharge of specified obligations of the Member such as the right to define the scientific programs or the obligation to provide parts of the contributions. Other fixed provisions regard reporting to the European Commission and to the ERIC Members.

To set-up an ERIC is simpler than for an IO. It requires (at least) three Countries to ask the EU Commission to set it up, with a draft Statute and a Technical Scientific document outlining scope and organisation. These are subjected to a first assessment allowing for clarifications and/or corrections (Step 1). The final proposal is then submitted formally to the Commission and its internal checks (Step 2). When approved it is published by the European Commission in the EU official Journal (with all required translations), and is immediately valid in all EU member Countries [7].

In absence of major disagreements, the whole procedure may last less than one year, compared to several years needed to achieve approval of an IO by several Parliaments.

\section{The evolving landscape of the ERICs}

In Table 1 there is a sample of physics-related or physics-intensive ERICs . More Physics-related ERICs are in the pipeline (e.g. ELI and CTAO [8]).

While previous international RIs were mainly large "one or few-sited" facilities, most of the new RIs respond to research needing either to collect geographically distributed data, or to integrate diverse techniques, or to access (remotely or physically) repositories of data, documents or samples: these are defined as "distributed". A good example is EPOS, allowing to access geophysical data in 256 smaller RIs covering most geophysical active areas in Europe. Access is through a single address and an integrated set of core services ensuring interoperability and compatibility of the data (Fig. 1, courtesy of EPOS-ERIC).

The concept of RI includes now instruments, collections and data banks distributed in several sites, and the access by external users can be "physical" (personal access), "remote" (sending samples, or remote control of instruments) or "virtual" (web-like access to data banks or computing facilities). The vocabulary has evolved in different ways in different RIs, local facilities being defined as "nodes", or "partner facilities" or "hubs" or "offices" ... etc. Many interesting facilities by different owners and their networking require sometimes a careful balance between coordination at national level and links at international level.

This variety may be complex, but a common element is a central governance/seat ensuring a joint planning of the resources and a "one stop" service to the external users. The need to offer to external users an effective point
14

\section{Europe needs excellent Research Infrastructures to compete with other large federated parts of the world 7 ?}

of access requires most distributed and multi-site RIs to integrate capabilities of different existing national or local facilities.

This stimulates management methods allowing a coherent activity between facilities in different countries under different legal and fiscal constraints. An effort to allow better employment for staff in different Countries is now ongoing. A new integrating pension scheme, RESAVER [9], for staff moving in Europe, has been set-up on proposal by EIROFORUM and ERF [10].

The implementation of the ERIC frame is still in its infancy, and several aspects are still being defined, from complete applicability of tax exemptions to full participation in internationally funded projects etc.. The impact of the ERIC on the European Research Area will make it a more structured and attractive space.

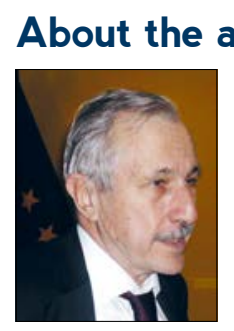

author

Carlo Rizzuto chairs the General Assembly of CERIC-ERIC. Researcher in basic and applied low temperature and materials sciences mainly at Genova University. Policy adviser in Italy and EU, implemented the Elettra Synchrotron, the FERMI FEL and INFM in Italy, and ESFRI in Europe.

\section{References}

[1] These are all well known in physics and direct info on their origin and institutional structure is available in their web sites

[2] ILL, the Institute Laue Langevin and ESRF, the European Synchrotron Radiation Facility, are at top world-level research infrastructures.

[3] Rossi G., Europhysics News 50/1, 14 (2019)

[4] https://eur-lex.europa.eu/legal-content/EN/TXT/?qid $=1464858864496 \&$ uri=CELEX\%3A32013R1261

[5] See also: https://ec.europa.eu/info/research-and-innovation/ strategy/european-research-infrastructures/eric/ eric-landscape_en

[6] https://ec.europa.eu/info/files/charter-access_en

[7] See https://op.europa.eu/en/publication-detail/-/publication/ c6647f05-874e-4cdd-af70-22ade4759930/language-en

[8] ELI and CTAO see details in the reference of note 8

[9] See http://www.RESAVER.eu

[10] EIROFORUM, is the Forum of the Intergovernmental and some of the "international Umbrella" organisations, see https://www.eiroforum.org/ ; ERF AISBL is the Association of international level Rls see https://erf-aisbl.eu/ 